\title{
Comparación de Controladores de Nivel para Canales Abiertos Basados en un Modelo por Colocación
}

\author{
Jean F. Dulhoste ${ }^{(1)}$, Didier Georges ${ }^{(2)}$, Gildas Besançon $^{(2)}$ y Carlos J. Jerez ${ }^{(1)}$ \\ (1) Universidad de Los Andes, Facultad de Ingeniería Mecánica, Departamento de \\ Ciencias Térmicas, Escuela de Ingeniería Mecánica, Mérida 5101-Venezuela \\ (e-mail: djean@ula.ve,rico@ula.ve) \\ (2) Instituto Politécnico Nacional de Grenoble-CNRS, Laboratorio de Automática de Grenoble-GSYS, \\ Saint Martin d'Hères-France (e-mail: didier.georges@inpg.fr, gildas.besancon@inpg.fr)
}

\section{Resumen}

El propósito del presente estudio es comparar el funcionamiento de controladores no lineales de nivel para canales abiertos. La dinámica del flujo en canales abiertos normalmente se representa mediante las ecuaciones de Saint-Venant. En este trabajo se presentan tres leyes de control no lineal que se desarrollaron a partir de un modelo de colocación. Las leyes incluidas son: linealización entrada salida, linealización entrada salida dinámica y linealización por realimentación escalonada (backstepping). Se implementó un simulador unidimensional para resolver las ecuaciones de SaintVenant, lo que permitió estudiar el comportamiento del sistema controlado. Los resultados de funcionamiento mostraron que la técnica de linealización entrada-salida dinámica parece ser la mejor de las tres estudiadas y que éstas técnicas muestran ser bastante precisas en los casos estudiados.

Palabras clave: canales abiertos, modelo de colocación, control no lineal, ecuaciones Saint-Venant

\section{A Comparative Study between Level Controllers for Open Channels Based on a Collocation Model}

\begin{abstract}
This paper proposed a comparative study between different nonlinear control techniques for level regulation in an open channel. Flow dynamics in a canal can be modeled by Saint-Venant equations. In this paper three control laws derived from a collocation model are presented. These laws are: input output linearization, dynamic input output linearization, and backstepping. A one-dimensional simulator that solves Saint-Venant's equations was implemented, allowing to study the controlled system. The results showed that the dynamic input output linearization control technique was the best among the studied control techniques, and that these techniques are reliable in the cases studied.
\end{abstract}

Keywords: open channel, nonlinear control, Saint-Venant equations, collocation method, input-output linearization 


\section{INTRODUCCIÓN}

En la actualidad, el control de un gran número de canales de irrigación se realiza operando manualmente las compuertas de regulación. En vista del mejoramiento de la gestión de dichos sistemas, se han realizado algunos trabajos con el objetivo de automatizar los canales de manera eficiente y segura. Para esto, el objetivo primordial es satisfacer la demanda de agua en cada estación de bombeo. Esto implica mantener un nivel de agua, que satisfaga la demanda sin permitir el desbordamiento del canal, para cada una de las secciones de canal y reservas existentes.

Entre los trabajos realizados al respecto, se pueden citar algunos basados en técnicas de control lineal tales como el uso de PID (Skertchly y Miles, 1994), control lineal cuadrático (Litrico y Georges, 2001; Malaterre, 1994), y control predictivo (Sawadogo, 1992). Los cuales, por utilizar técnicas lineales, son válidos solo alrededor de un punto de operación. Otros, utilizan técnicas no lineales, basadas en las ecuaciones de Saint-Venant (Graf y Altinakar, 2000), usando métodos de control óptimo no lineal (Ding y Wang, 2006) o mediante un método basado en los invariantes de Reinmann (Halleux et al., 2003). Estos métodos son difícilmente aplicables a un canal real por su complejidad.

Uno de los problemas que se observa, es la necesidad de obtener un modelo simple, que mantenga la información proveniente de los fenómenos no lineales presentes en el sistema. Para esto, existen varios métodos que permiten simplificar las ecuaciones de Saint-Venant, entre los que se pueden citar: diferencias finitas (Strelkoff, 1970), elementos finitos (Colley y Moin, 1976), y el método de colocación por puntos (Dulhoste et al., 2004). De estos métodos, el último, que es un caso especial de los métodos de residuos ponderados (Fletcher, 1984), es muy utilizado en sistemas químicos o físicos (Dochain et al., 1992), pero muy poco en sistemas hidráulicos de canales abiertos. En artículos anteriores, se mostró que este método puede ser utilizado como modelo de base para el control de canales abiertos. En Dulhoste et al. (2004) se presenta un control no lineal basado en técnicas de linealización entrada-salida (Isidori, 1995) y linealización entrada-salida dinámica, y en Besançon et al. (2001), se presenta un control basado en técnicas de backstepping (Krstic et al., 1995).

El objetivo de este trabajo es presentar un resumen comparativo de estos controladores no lineales, desarrollados en detalle en trabajos anteriores (Dulhoste et al., 2004; Besançon et al., 2001). El presente artículo está organizado como sigue: En la sección 2 se incluye un breve resumen del modelo utilizado para el control, en la sección 3 se incluye un resumen de los tres controladores a comparar, la sección 4, que es el aporte principal del artículo, trata sobre la comparación de los tres métodos mediante simulaciones realizadas en condiciones similares, y finalmente la sección 5 incluye las conclusiones del trabajo.

\section{MODELO DE LA DINÁMICA DE FLUJO EN CANALES ABIERTOS}

\section{Ecuaciones de Saint-Venant}

El modelo, comúnmente utilizado para representar el flujo en canales abiertos, es un sistema de ecuaciones diferenciales parciales no lineales, denominadas ecuaciones de Saint-Venant:

$$
\begin{aligned}
& \frac{\partial S}{\partial t}+\frac{\partial Q}{\partial x}=q \\
& \frac{\partial Q}{\partial t}+\frac{\partial\left(Q^{2} I S\right)}{\partial x}+g S\left(\frac{\partial h}{\partial x}-I+J\right)=k_{q} q \frac{Q}{S}
\end{aligned}
$$

Donde $J=Q|Q| \imath\left(k S(S / p)^{2 / 3}\right)^{2}$ : fricción; $k$ : Coeficiente de Strickler; $S$ : sección mojada; $Q$ : flujo o gasto de agua; $h$ : nivel relativo al fondo; $l$ : pendiente del fondo; $q$ : infiltración; $K_{q}=0$ si $q>0$, y $K_{q}=1$ si $q<0$. Considerando el caso de un canal de sección rectangular se tiene que $S=B h$ y $p=B+2 h$, donde $B$ y $L$ son el ancho y el largo del canal respectivamente. Para un canal fluvial (flujo subcrítico) se debe 
usar una condición de frontera aguas arriba y otra aguas abajo, se escogió el flujo en las dos compuertas, utilizado luego como variable de control:

$Q_{(x=0, t)}=Q_{0(t)} ; \quad Q_{(x=L, t)}=Q_{n(t)}$

Finalmente se deben tomar en cuenta las condiciones iniciales $h(x, 0)=h_{l}(x) ; \quad Q(x, 0)=Q_{l}(x)$; $\forall x \in[0, L]$.

Modelo simplificado de las ecuaciones de Saint-Venant basado en el método de colocación

En Dulhoste et al. (2004), se mostró que era posible simplificar las ecuaciones diferenciales parciales de Saint-Venant, transformándolas en un sistema de ecuaciones diferenciales ordinarias para cada punto de colocación, así para tres puntos y las condiciones de frontera de la Ec. (2), se obtiene:

$\left\{\begin{array}{l}\dot{h}_{1}=\frac{1}{B L}\left[-4 Q_{2}+3 u_{1}+u_{2}\right]+\frac{q}{B} \\ \dot{h}_{2}=\frac{1}{B L}\left[u_{1}-u_{2}\right]+\frac{q}{B} \\ \dot{h}_{3}=\frac{1}{B L}\left[4 Q_{2}-u_{1}-3 u_{2}\right]+\frac{q}{B} \\ \dot{Q}_{2}=a\left(Q_{2}, h_{2}\right)\left(h_{3}-h_{1}\right)+f\left(Q_{2}, h_{2}, u_{1}, u_{2}, q\right)\end{array}\right.$

Donde $u_{1}$ y $u_{2}$ representan las variables de control (flujo en los extremos), $Q_{2}$ el caudal en centro y:

$a\left(Q_{2}, h_{2}\right)=\left(\frac{Q_{2}{ }^{2}}{B L h_{2}{ }^{2}}-\frac{g B h_{2}}{L}\right) ; f\left(Q_{2}, h_{2}, u_{1}, u_{2}, q\right)=-\frac{g Q_{2}{ }^{2}}{k^{2} B h_{2}\left(B h_{2} l\left(B+2 h_{2}\right)\right)^{4 / 3}}+\left(\frac{2\left(u_{1}-u_{2}\right)}{B L}+\frac{k_{q} q}{B}\right) \frac{Q_{2}}{h_{2}}+g B h_{2} I$

\section{LEYES DE CONTROL}

En esta sección se resumen las leyes de control desarrolladas en trabajos anteriores y utilizados aquí para la comparación. Las tres leyes presentadas tienen por objetivo el control de nivel en las extremidades del canal, con $y_{1}$ y $y_{2}$ son las salidas y $h_{\mathrm{e}}$ el nivel deseado. Se escoge como salidas:

$y=\left(\begin{array}{l}y_{1} \\ y_{2}\end{array}\right)=\left(\begin{array}{l}h_{1}-h_{e} \\ h_{3}-h_{e}\end{array}\right)$

Control por linealización entrada salida

En Dulhoste et al. (2004) se mostró que si el sistema satisface la condición:

$\forall t \geq 0, \quad y_{2}(t)-y_{1}(t)<\min \left(L I, \frac{g L}{k^{2} B}\right)$

Entonces el controlador de la Ec.(7) garantiza que el sistema a lazo cerrado es estable con $\lim _{t \rightarrow \infty} h_{1}(t)=\lim _{t \rightarrow \infty} h_{3}(t)=h_{e}$ para todo $Q_{2}(0) \geq 0$.

$u=M^{-1}\left[-K_{1}\left[\begin{array}{l}h_{1}-h_{e} \\ h_{3}-h_{e}\end{array}\right]-\frac{Q_{2}}{B L}\left[\begin{array}{c}-4 \\ 4\end{array}\right]-\frac{q}{B}\left[\begin{array}{l}1 \\ 1\end{array}\right]\right]$ 
Donde la matriz diagonal $K_{1}>0$ y $M=\frac{1}{B L}\left[\begin{array}{cc}3 & 1 \\ -1 & -3\end{array}\right]$

\section{Control por linealización entrada salida dinámica}

En Dulhoste et al. (2004) se mostró que si el sistema satisface la condición expresada en la Ec. (6), entonces el controlador dinámico de la Ec. (8) garantiza que el sistema a lazo cerrado es estable con $\lim _{t \rightarrow \infty} h_{1}(t)=\lim _{t \rightarrow \infty} h_{3}(t)=h_{e}$ para todo $Q_{2}(0) \geq 0$.

$\dot{u}=\left[\frac{2 Q_{2}}{B L h_{2}}\left[\begin{array}{ll}1 & -1 \\ 1 & -1\end{array}\right]-M^{-1} K_{1} M\right] u-M^{-1}\left[\frac{4}{B L}\left(\left(Q_{2} K_{1}+F 1\right)\left[\begin{array}{c}-1 \\ 1\end{array}\right]\right)+K_{1} \frac{q}{B}\left[\begin{array}{l}1 \\ 1\end{array}\right]+K_{2}\left(\begin{array}{l}h_{1}-h_{e} \\ h_{3}-h_{e}\end{array}\right)\right]$

Donde $K_{1}>0, K_{2}>0 \mathrm{y}$ :

$F 1=\left(\frac{Q_{2}{ }^{2}\left(-h_{1}+h_{3}\right)}{L B h_{2}{ }^{2}}+\frac{k_{q} q}{B} \frac{Q_{2}}{h_{2}}-g B h_{2}\left[\frac{\left(-h_{1}+h_{3}\right)}{L}-I+\frac{Q_{2}{ }^{2}}{k^{2} B^{2} h_{2}{ }^{2}\left(B h_{2} I\left(B+2 h_{2}\right)\right)^{4 / 3}}\right]\right)$

\section{Control por Backstepping}

En Besançon et al. (2001), se mostró que haciendo el cambio de variable: $U_{1}=u_{1}-u_{2}, U_{2}=u_{1}+u_{2}$, $h_{3}-h_{1}=H$, y bajo la condición $a\left(Q_{2}, h_{2}\right) \neq 0$, el controlador de la Ec. (10) garantiza que el sistema en lazo cerrado es estable con $\lim _{t \rightarrow \infty} h_{1}(t)=\lim _{t \rightarrow \infty} h_{3}(t)=h_{e}$ para todo $Q_{2}(0)$.

$U_{1}=-B L K_{1}\left(h_{2}-h_{2 e}\right)-L q$

$U_{2}=\frac{B L}{4} K_{22} H_{d}+2 Q_{2}+a\left(Q_{2}, h_{2}\right) \frac{B L}{4} Q_{d}+\frac{B L}{4} \frac{d}{d t}\left(H_{e}\right)$

Donde $Q_{d}=Q_{2}-Q_{2 e} ; H_{d}=H-H_{e}$

$$
Q_{d}=Q_{2}-Q_{2 e} ; H_{d}=H-H_{e} ; H_{e}=-\frac{\left[K_{21}\left(Q_{d}\right)+f\left(Q_{2}, h_{2}, U_{1}, q\right)\right]}{a\left(Q_{2}, h_{2}\right)} \text { y } h_{2 e}=\frac{2 h_{e}-h_{1}(0)-h_{3}(0)+2 h_{2}(0)}{2}
$$

y $Q_{2 e}$ es raíz de $H_{e}\left(Q_{2}, h_{2 e}\right)=0$. Siendo los coeficientes $K_{1}, K_{21}$ y $K_{22}$ positivos.

\section{COMPARACIÓN DE LOS CONTROLADORES}

Los controladores no lineales antes presentados, provienen del mismo modelo matemático, obtenido por un método de colocación, sin embargo presentan características particulares, las cuales pueden observarse en simulaciones, o directamente en el modelo. En cuanto a las diferencias observables directamente en el modelo están las condiciones de estabilidad para cada uno de estos controladores. En este sentido, la estabilidad de los controladores obtenidos por linealización entrada-salida está garantizada sólo para flujos iniciales positivos, con una pendiente de la superficie del agua que cae hacia la zona aguas abajo. Mientras que el controlador obtenido por backstepping es estable para cualquier flujo inicial, siempre y cuando $a \neq 0$, nótese que $a=0$ solo para un flujo crítico (número de Froude igual a 1). Para ilustrar las diferencias que se observan en la simulación se presenta a continuación una serie de figuras donde se utiliza un canal de $1000 \mathrm{~m}$ de largo, $1 \mathrm{~m}$ de ancho, pendiente de $0.1 \%$ y coeficiente de Strickler de 25 . Se somete este canal a un cambio del 
nivel de referencia, pasando este de 1.5 a $1.8 \mathrm{~m}$. Para estudiar el comportamiento del sistema controlado, se escogió para el canal un modelo más preciso que el obtenido por el método de colocación. Se utilizó un modelo de diferencias finitas implícito, conocido como modelo de Preissman (Malaterre, 1994).

La Fig. 1 presenta las simulaciones realizadas con este modelo. Se observa en estas simulaciones que los dos controladores por linealización entrada salida dan resultados similares, siendo la respuesta del dinámico un poco más rápida, pero el controlador por Backstepping presenta oscilaciones fuertes que tienden a desestabilizarlo, de hecho la simulación fue cortada antes de aparecer las fuertes oscilaciones. Esto indica, que este último control no es robusto, respecto al modelo usado en la simulación, por lo que se puede presumir que será difícil usarlo en un sistema real.

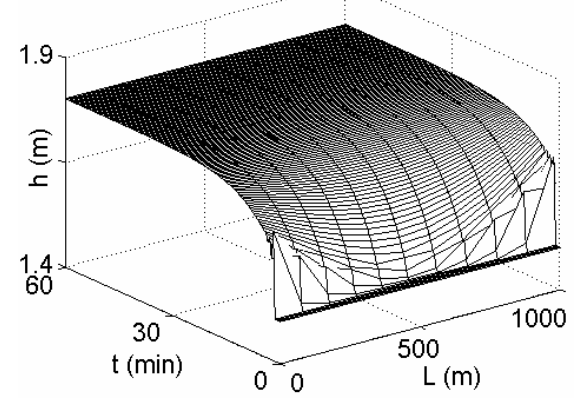

a) Linealización E.S



b) Linealización E.S.D.

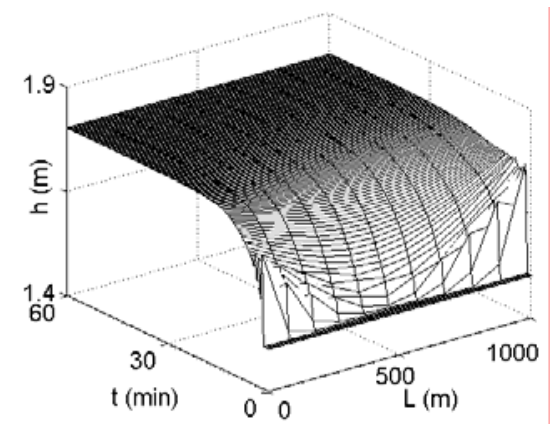

c) Backstepping

Fig. 1: Evolución del nivel en canal de 1000 m con modelo de simulación de Preissman con 10 secciones

La Fig. 2 muestra simulaciones similares para un canal de $5 \mathrm{~km}$ y 20 secciones. En estas se observa que el controlador por linealización entrada salida presenta unas oscilaciones muy fuertes al principio de la simulación, oscilaciones que son mucho más suaves en el controlador dinámico. El controlador Backstepping no funcionó para esta longitud. Esto nos indica que el primero es menos robusto para canales largos. De hecho, en simulaciones para canales más largos, se observa que estas oscilaciones aumentaban hasta el punto de volver inestable el sistema, y para disminuir estas oscilaciones era necesario aumentar considerablemente el número de secciones del simulador.



a) Linealización E.S.



b) Linealización E.S.D

Fig. 2: Evolución del nivel en canal de 5 km con modelo de simulación de Preissman con 10 secciones

\section{CONCLUSIONES}

Se puede decir que de los tres controladores comparados, el controlador elaborado con métodos de linealización entrada-salida dinámica parece ser el más rápido y robusto, siendo los tres bastante 
precisos en los casos donde funcionan. Esto a pesar de que teóricamente el controlador por backstepping parece ser mejor ya que su estabilidad esta garantizada en un rango de funcionamiento más amplio, pero las simulaciones con el modelo de diferencias finitas mostró que esto es válido solo para el modelo de colocación a 3 puntos.

Entre las perspectivas de este trabajo, a futuro, está el probar estas leyes de control en un banco de pruebas real, compararlas con otras leyes existentes y probar su funcionamiento en presencia de un observador del flujo del canal, este último para poder controlar el canal con solo medidas de nivel.

\section{REFERENCIAS}

Besançon, G., J.F. Dulhoste y D. Georges; A Nonlinear Backstepping Like Controller for a ThreePoint Collocation Model of Water Flow Dynamics. Conference on Control Application, México (2001).

Colley, R.L. y S.A. Moin; Finite Element Solution of Saint-Venant Equations. Journal of Hydraulical Engineering. Division ASCE: 102(HY6), 759-775 (1976).

Ding, Y. y S.S.Y. Wang; Optimal Control of Open-Channel Flow Using Adjoint Sensitivity Analysis. ASCE, Journal of Hydraulic Engineering: 132(11), 1215 - 1228 (2006).

Dochain, D., J.P. Babary y N. Tali-Maamar; Modeling and Adaptative Control of Nonlinear Distributed Parameter Bioreactor via orthogonal collocation. Automatica: 28(5), 873-883 (1992).

Dulhoste J.F, D. Georges G. y Besançon; Nonlinear Control of Open Channel Water Flow Based on a Collocation Control Model, Journal of Hydraulic Engineering. Division ASCE:130(3), 254-266 (2004).

Fletcher, C.A.J.; Computational Galerkin Methods. Springer-Verlag. New York USA (1984).

Graf, W.H. y M. Altinakar ; Hydraulique Fluviale, $2^{\text {a }}$ edición. Presses Polytechniques et Universitaires Romandes, Lausanne-Switzerland (2000).

Halleux, J. y otros cuatro autores; Boundary Feedback Control in Networks of Open Channels. Automatica: 39(8), 1365 - 1376 (2003).

Isidori, A.; Nonlinear Control Systems, $3^{a}$ edición. Springer-Verlag, Berlin-Germany (1995).

Krstic, M., I. Kanellakopoulos y P. Kokotovic; Nonlinear and Adaptive Control Design; Wiley Interscience, New York-USA (1995).

Litrico, X. y D. Georges; Robust LQG control of single input multiple output dam-river systems. International Journal of Systems Science: 32(6), 798-805. (2001).

Malaterre, P.O.; Modèlisation, Analyse et Commande Optimale LQR d'un Canal d'Irrigation. Ph.D. thesis. ISBN 2-85362-368-8. LAAS-CNRS-ENGREF-Cemagref (1994).

Sawadogo, S.; Modelisation, Commande Predictive et Supervision d'un Système d'Irrigation. Informe de tésis de doctorado. Université Paul Sabatier. Toulouse-France (1992).

Skertchly, L. y J.P. Miles; Control of Irrigation Canal. Journal of Hydraulic Engineering. Division ASCE: 122(7), 403-410 (1994).

Strelkoff, T.; Numerical Solution of Saint-Venant Equation. Journal of Hydraulic Engineering. Division ASCE: 96(HY1), 223-252 (1970). 